\title{
Flocculated flow of microfibrillated cellulose water suspensions: an imaging approach for characterisation of rheological behaviour
}

\author{
Eve Saarikoski · Tapio Saarinen • Juha Salmela • \\ Jukka Seppälä
}

Received: 3 November 2011 / Accepted: 2 February 2012/Published online: 22 February 2012

(C) Springer Science+Business Media B.V. 2012

\begin{abstract}
Our aim was to characterise the suspension rheology of microfibrillated cellulose (MFC) in relation to flocculation of the cellulose fibrils. Measurements were carried out using a rotational rheometer and a transparent cylindrical measuring system that allows combining visual information to rheological parameters. The photographs were analyzed for their floc size distribution. Conclusions were drawn by comparing the photographs and data obtained from measurements. Variables selected for examination of MFC suspensions were degree of disintegration of fibres into microfibrils, the gap between the cylinders, sodium chloride concentration, and the effects of changing shear rate during the measurement. We studied changes in floc size under different conditions and during network structure decomposition. At rest, the suspension consisted of flocs sintered together into a network. With shearing, the network separated first into chain-like floc formations and, upon further shear
\end{abstract}

Eve Saarikoski and Tapio Saarinen have contributed equally to this work.

E. Saarikoski · T. Saarinen · J. Seppälä $(\bowtie)$

School of Chemical Technology, Polymer Technology,

Aalto University, P.O. Box 16100, 00076 Aalto, Espoo,

Finland

e-mail: jukka.seppala@aalto.fi

J. Salmela

VTT Processes, Koivurannantie 1, P.O. Box 1603, 40101 Jyväskylä, Finland rate increase, into individual spherical flocs. The size of these spherical flocs was inversely proportional to the shear rate. Investigations also confirmed that floc size depends on the geometry gap, and it affects the measured shear stress. Furthermore, suspension photographs revealed an increasing tendency to aggregation and wall depletion with sodium chloride concentration of $10^{-3} \mathrm{M}$ and higher.

Keywords Microfibrillated cellulose $\cdot$ Water suspension · Flocculation · Rheology $\cdot$ Image analysis

\section{Introduction}

Microfibrillated cellulose (MFC) water suspensions have been investigated since the 1980s when Turbak et al. (1983) and Herrick et al. (1983) reported a route for defibrillation of cellulose into its constituent components and suggested very promising applications in thickening foodstuff and suspending solids. The manufacturing of MFC was, however, prohibitively expensive on an industrial scale due to availability of only energy intensive process technologies. Since the 1990s, great progress has been made towards finding economically feasible ways of producing MFC, including enzymatic pretreatment of the pulp (Henriksson et al. 2007), carboxymethylation (Wågberg et al. 1987; Saito et al. 2006), and TEMPO mediated oxidation of the fibres (Saito et al. 2006) followed by less intensive mechanical fibre disintegration. 
New applications, besides using MFC in suspension form, have been proposed in composites utilizing the transparency of the most defibrillated MFC grades (Ono et al. 2001; Ono et al. 2004; Saito et al. 2006) and the low thermal expansion of fibrils in e.g. optoelectronics (Yano et al. 2005). Even for these purposes, the manufacturing still includes steps in which the fibrils are in a suspension. Therefore, understanding how to control the rheological behaviour of MFC suspensions is crucial for fully exploiting the capabilities of the material.

Several rheological variables have already been studied with different types of MFC suspensions. The suspensions are usually utilised in concentrations where they form a gel network at rest, i.e. the rheological response of the suspension is elastic dominated (storage modulus $\mathrm{G}^{\prime}>$ loss modulus $\mathrm{G}^{\prime \prime}$ ) and largely independent of reference time (angular frequency $\omega$ ). The critical concentration for gel formation is inversely proportional to fibre axial ratio a/l (diameter to length) (Tatsumi et al. 2002; Hill 2008; Lasseuguette et al. 2008). Furthermore, the rigidity of the elastic gel network at rest $\left(\mathrm{G}^{\prime}\right.$, from a frequency sweep spectrum) above the percolation limit is scaled following the power law (Tatsumi et al. 2002; Pääkkö et al. 2007; Hill 2008; Agoda-Tandjawa et al. 2010), $G^{\prime} \propto k \varphi^{a}$, where the factor $\mathrm{k}$ depends on axial ratio (Tatsumi et al. 2002), $k \propto(a / l)^{2}$, and exponent a on fibril volume fraction (Hill 2008), $a \propto \varphi$.

Under steady shear conditions, MFC suspensions are both shear thinning and thixotropic (Turbak et al. 1983; Goussé et al. 2004; Pääkkö et al. 2007; Lasseuguette et al. 2008; Agoda-Tandjawa et al. 2010), due to the prevailing fibril organisation being broken down by shearing and the time it takes to reach an equilibrium at any given shear rate. The flow curve of MFC suspensions has a characteristic shape. At low shear rates, the shear stress is constant, however, above a certain threshold the shear stress rises as a function of shear rate (Pääkkö et al. 2007; Hill 2008; Lasseuguette et al. 2008; Agoda-Tandjawa et al. 2010). The plateau region at low shear rates is a measure of yield (Tatsumi et al. 2002; Agoda-Tandjawa et al. 2010). The rise in shear stress at a higher shear rate may be due to the organisation of fibrils into a flowing formation (Agoda-Tandjawa et al. 2010; Iotti et al. 2011).

The above mentioned rheological MFC suspension studies mainly dealt with fibre network characteristics, and the way in which the fibrils are organised within the network, i.e. in flocs, has received only limited attention (Tatsumi et al. 2002; Ono et al. 2004; Hill 2008; Lasseuguette et al. 2008). Studies performed with both macroscopic fibre suspensions (Björkman 2000; Karema et al. 2001; Chen et al. 2002; Björkman 2005; Salmela and Kataja 2005; Hubbe 2007) and micro scale colloidal suspensions (Hubbe and Rojas 2008; Mewis and Wagner 2009a), however, show the great significance of flocculation and the need to analyse its contribution to suspension characteristics. Flocculation affects rheological properties of the suspensions in both processing and at rest. Furthermore, the quality of the products is also dependent on the homogeneity of the fibril distribution (Björkman 2006; Hubbe 2007), e.g. paper, films, and composites.

MFC suspensions have both colloidal and noncolloidal characteristics. In MFC the diameter of fibrils depends on the fibril source, their pre-treatment and the degree of fibrillation. The smallest diameters start from 2 to $5 \mathrm{~nm}$ of single cellulose I fibrils ranging up to several tens of nanometers of fibril aggregates (Pääkkö et al. 2007; Agoda-Tandjawa et al. 2010). These values are well below the $1 \mu \mathrm{m}$ order where colloidal phenomena start to dominate over gravity and inertia in the rheological characteristics (Hubbe and Rojas 2008). Flocculation of fibres can be divided into two types, macroscopic- and molecular scale (Hubbe 2007). On a macroscopic scale, it is related to fibre content of the suspension and to fibres gyration when the suspension is stirred, which leads fibres to collide, entangle, and flocculate. On a molecular scale, it is related to colloidal interactions between particles approximately $1-100 \mathrm{~nm}$ from each other. These interactions are related to the electrostatic and dispersion effects between fibrils. Both macroscopic and microscopic flocculation factors play a role in microfibrillated cellulose suspensions, but which is dominant is a balance between fibre dimensions and surface properties.

The reason flocculation has received minimal attention in the MFC suspension rheology is, conventional rheological experiments do not provide visual information on the state of the fibrils during the experiment. Therefore, given this restraint, one has been left to ponder with secondary and often very conflicting data on the contribution of flocs (Abe et al. 2007). With macroscopic cellulose suspensions, imaging studies have been conducted by several authors 
(e.g. Björkman 2000; Karema et al. 2001; Chen et al. 2002; Björkman 2005; Salmela and Kataja 2005). To bridge the gap to microscopic scale, we took a new transparent, cylindrical measuring system into use. This allows us to combine visual information with rheological parameters and see how the floc structure adapts to the varying shear conditions. We show how the degree of disintegration of fibres into microfibrils, the gap between the cylinders, sodium chloride concentration, and the effects of changing shear rate during the measurement are reflected in the floc structure and the suspension rheology.

\section{Materials and methods}

The starting material was never dried pre-refined birch pulp. First, the pulp was sodium-washed, diluted to $1 \%$ wt and dispersed for $40 \mathrm{~min}$ at 2,400 rpm with a Dispermat TU laboratory dissolver (VMA-Getzmann GMBH, Germany). The disintegration of fibres into microfibrils was then executed with Microfluidics ${ }^{\circledR}$ M110Y Microfluidizer (Microfluidics International Corporation, USA), a high pressure fluids processor. The first fluidisation pass was through 400 and $200 \mu \mathrm{m}$ chambers with a 900 bar pressure, and the following three or four passes through 200 and $100 \mu \mathrm{m}$ chambers at a pressure of 1,300 bar. Resulting MFC suspensions 1 and 2 are presented in Table 1 . The total number of passes through the fluidizer was determined based on preliminary tests to obtain floc structure sizes in the observable scale.

$\mathrm{NaCl}$ containing MFC suspensions were also prepared in order to estimate the effects of ionic strength on the flocculation and flow properties. $\mathrm{NaCl}$ concentrations varied from $10^{-4}$ to $1 \mathrm{M}$, as shown in

Table 1 MFC suspensions used in the study

\begin{tabular}{lll}
\hline Suspension & $\begin{array}{l}\text { Number of } \\
\text { fluidisation passes }\end{array}$ & $\begin{array}{l}\mathrm{NaCl} \text { concentration } \\
(\mathrm{mol} / \mathrm{dm} 3)\end{array}$ \\
\hline 1 & 4 & - \\
2 & 5 & - \\
3 & 5 & $10-4$ \\
4 & 5 & $10-3$ \\
5 & 5 & $10-2$ \\
6 & 5 & $10-1$ \\
7 & 5 & 1 \\
\hline
\end{tabular}

The MFC concentration of every sample was $1.0 \% \mathrm{wt}$
Table 1. To mix in the salt the suspensions went through the fluidiser once more, but this time only through the $400 \mu \mathrm{m}$ chamber to prevent further fibre defibrillation. The unsalted, five times fluidised suspension also received the same final treatment in order to set an equal shear history. After the defibrillation the suspensions were stored in a refrigerator. The samples were loaded into the rheometer with a stainless steel spoon preceded by gentle stirring to mix in the diminutive amount of water separated during the storage.

Rheological characterisation

All the rheological measurements were recorded using the Physica MCR 301 rotational rheometer (Anton Paar GmbH, Austria) equipped with concentric cylinders geometries and a camera for taking images, see Fig. 1. As a reference point, DIN concentric cylinders geometry was used with bob and stainless steel cup diameters of 26.67 and $28.92 \mathrm{~mm}$. To facilitate imaging, two simple glass outer cylinder geometries were assembled at our laboratory, see Table 2 and Fig. 2. The measurements were performed at room temperature $\left(23-25^{\circ} \mathrm{C}\right)$.

In order to homogenise the floc structure and set a comparable shear history for each sample a preshear interval of $25 \mathrm{~min}$ with a shear rate $(\dot{\gamma})$ of $200 \mathrm{~s}^{-1}$ was performed at the start of the rheological measurements. Furthermore, during this period the bulk of air bubbles trapped in the suspension were relieved. After the preshear, the suspension was left to recover for $1 \mathrm{~h}$,

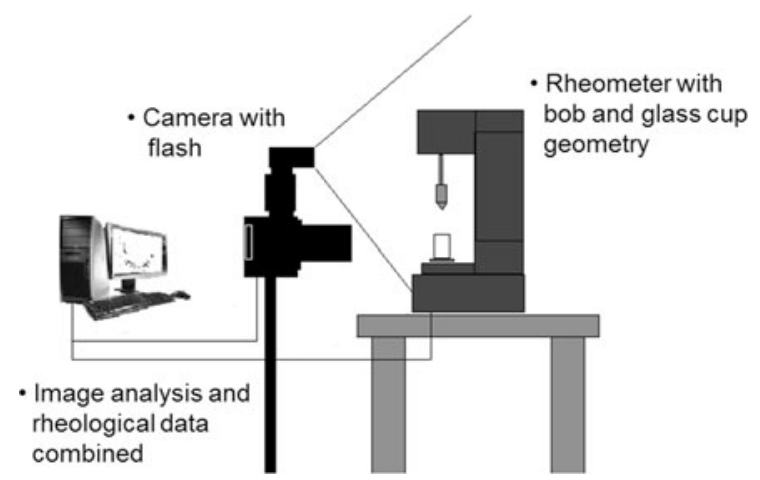

Fig. 1 A schematic representation of the equipment used for flow of MFC suspension observations. The equipment consists of a camera and rheometer with a transparent outer geometry. The rheological data and images are combined after the measurement 
Table 2 Cup diameters for stainless steel and glass cups

\begin{tabular}{lll}
\hline Cup & Diameter $(\mathrm{mm})$ & Gap $(\mathrm{mm})$ \\
\hline Glass 1 & $28.53 \pm 0.08$ & $0.93 \pm 0.08$ \\
Glass 2 & $29.21 \pm 0.036$ & $1.27 \pm 0.04$ \\
Stainless steel & 28.92 & 1.13 \\
\hline
\end{tabular}

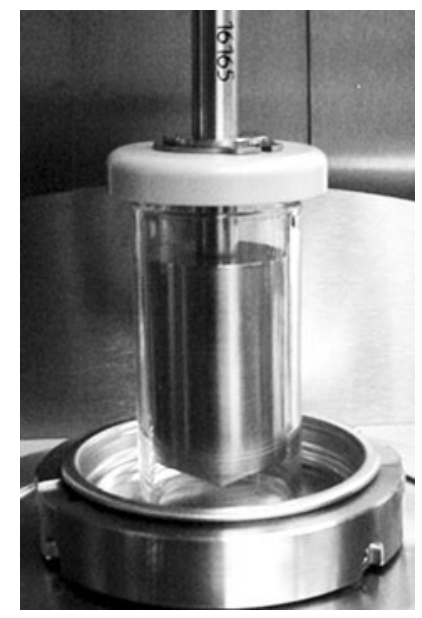

Fig. 2 View of the geometry used in the measuring position

under small amplitude oscillatory shear (angular frequency $(\omega) 6.28 \mathrm{rad} / \mathrm{s}$, strain $1 \%$, i.e. well within the linear viscoelastic region). During the recovery period, flocculated fibre network development was observed by storage and loss moduli values $\left(\mathrm{G}^{\prime}\right.$ and $\mathrm{G}^{\prime \prime}$ ) together with floc growth. After the recovery period, the breakdown of the flocculated fibre network was followed in a series of peak hold experiments. The shear rates used in the peak hold experiments varied between 1 and $50 \mathrm{~s}^{-1}$ with each step lasting $10 \mathrm{~min}$.

Image analysis

The photographs were shot with a Nikon D90 DSLR camera with an AF Micro NIKKOR $60 \mathrm{~mm} \mathrm{1:2,} 8 \mathrm{D}$ macro lens and Nikon Speedlight SB-800 flash. Images were taken using ISO 200 sensitivity and lens apertures f36/f40. The use of a small aperture was enabled by targeting additional lighting at $90^{\circ}$ to the subject and setting a white background paper behind the geometry. Focusing was manual to a point marked inside the glass geometry. The point was erased before metering out the suspension.
Floc size analysis was performed using a method presented by Karema et al. (2001). Uneven illumination in each image was corrected for using an average image of all acquired images. This correction removes all stationary light intensity variations from original images. For this reason all remaining intensity variations in corrected images are due to changes in suspension properties. Dominant factor causing most of these variations is suspension local concentration. To determine the floc size from the enhanced images, a threshold operation with the median of intensity was carried out in a way that $50 \%$ of the image area was marked as flocs and $50 \%$ as voids. The distribution of floc dimensions in both $\mathrm{x}$ and $\mathrm{y}$ directions were then computed for every image in the sequence as the run-length distributions $\hat{f}\left(\mathrm{~L}_{\mathrm{x}}\right)$ and $\hat{f}\left(\mathrm{~L}_{\mathrm{y}}\right)$. (The run length $\mathrm{L}_{\mathrm{i}}$ is defined as the maximum length of continuous line segment in direction $i=x, y$ that is entirely within the 'floc region' at given location in the threshold image). These distributions were finally averaged to produce the smooth distributions $f(L x)$ and $f(L y)$ shown in Fig. 3.

\section{Results and discussion}

First, we will discuss what happens to the suspension structure during the preshear and subsequent recovery intervals. We will then follow the suspension structure decomposition upon introducing steady shear flow conditions. We will accomplish this by investigating a series of linked peak hold experiments. Next, we will show how the floc size and rheology of the suspensions is dependent on the measuring geometry gap and material. We will further demonstrate the effect of the degree of fibrillation on the floc size by comparing four and five times fluidised samples. Finally, we will discuss how adding $\mathrm{NaCl}$ to adjust the suspension ionic strength changes the rheology.

\section{Preshear and recovery periods}

Apart from fibrils characteristics, the structure of flocculated fibre network is dependent on time and the shear conditions. Under shear, the suspension undergoes fibril network disintegration along with dynamic reconstruction of the network, both dependent on collisions of fibres by shearing and the Brownian motion (Barnes 1997; Mewis and Wagner 2009b). 
Fig. 3 Sequence of illumination correction for one image and resulting floc size distribution
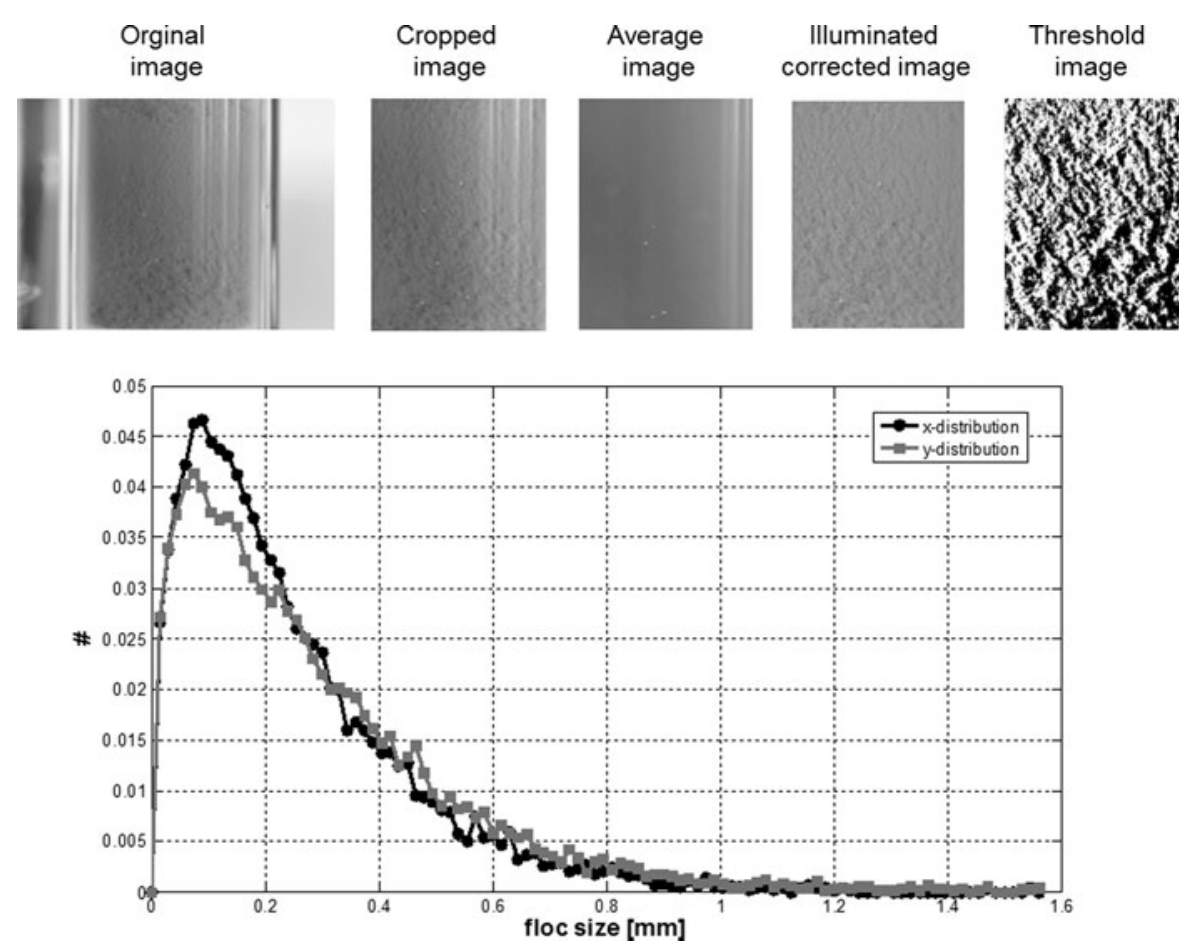

Depending on the shear rate, individual fibrils move between the flocs while the floc structure adapts to the prevailing shear conditions. During the preshear period steady shear flow conditions at $200 \mathrm{~s}^{-1}$, the suspension structure approaches steady floc size. This can be inferred from the shear stress leveling of five times fluidised sample in Fig. 4 and the fact that under such high shear rate, structure evolves rather fast in relation 0 to the $10 \mathrm{~min}$ observation period. Under these conditions, there is no time for contact point optimisation between individual, flocculated fibrils compared to the fast macroscopic, hydrodynamic interactions between flocs, which are due to physical collisions, and result in the adaptation of the floc structure to the prevailing shear conditions.

When shearing is stopped, the structure starts to recover immediately. The apparent floc structure freezes and the suspension retains the macroscopic floc size and the floc size distribution formed during the previous shear interval (Fig. 5a). Despite the constant apparent floc size during the recovery, the moduli levels rise significantly over the time (Fig. 7). At rest, the fibrils are limited to local motion, and the rises in the moduli levels reflects the optimisation of fibril contact points, both intra and inter flocs, while the flocs are being sintered together.

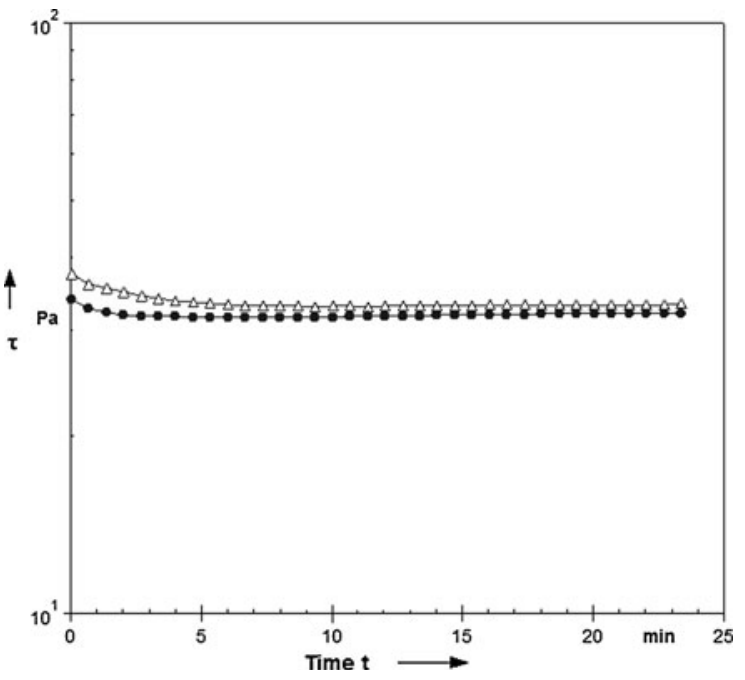

Fig. 4 The leveling of shear stress as a function of time during the preshear interval. The geometries in use were stainless steel (gap $1.1 \mathrm{~mm}$, filled circle) and glass 2 (gap $1.3 \mathrm{~mm}$, open triangle $)$ and the sample was fluidised five times

Steady shear intervals

After the recovery period, upon introducing a constant shear rate in a series of peak hold experiments, the flocculated fibril network starts to deform and finally 
Fig. 5 Different stages in flocculated flow at several shear rates for five times fluidised MFC suspension a at rest after the $25 \mathrm{~min}$ preshear interval at $200 \mathrm{~s}^{-1}$. The floc structure stems from the preshear period, b $1 \mathrm{~s}^{-1}$, flocs start to move in stress chains, $5 \mathrm{~s}^{-1}$, chain compressing, d $10 \mathrm{~s}^{-1}$, chain splitting, e $50 \mathrm{~s}^{-1}$, flocculated flow of individual flocs. A picture of each state was taken after shearing $9 \min 55 \mathrm{~s}$ at the indicated shear rate with the exception of a which was taken after $1 \mathrm{~h}$ recovery

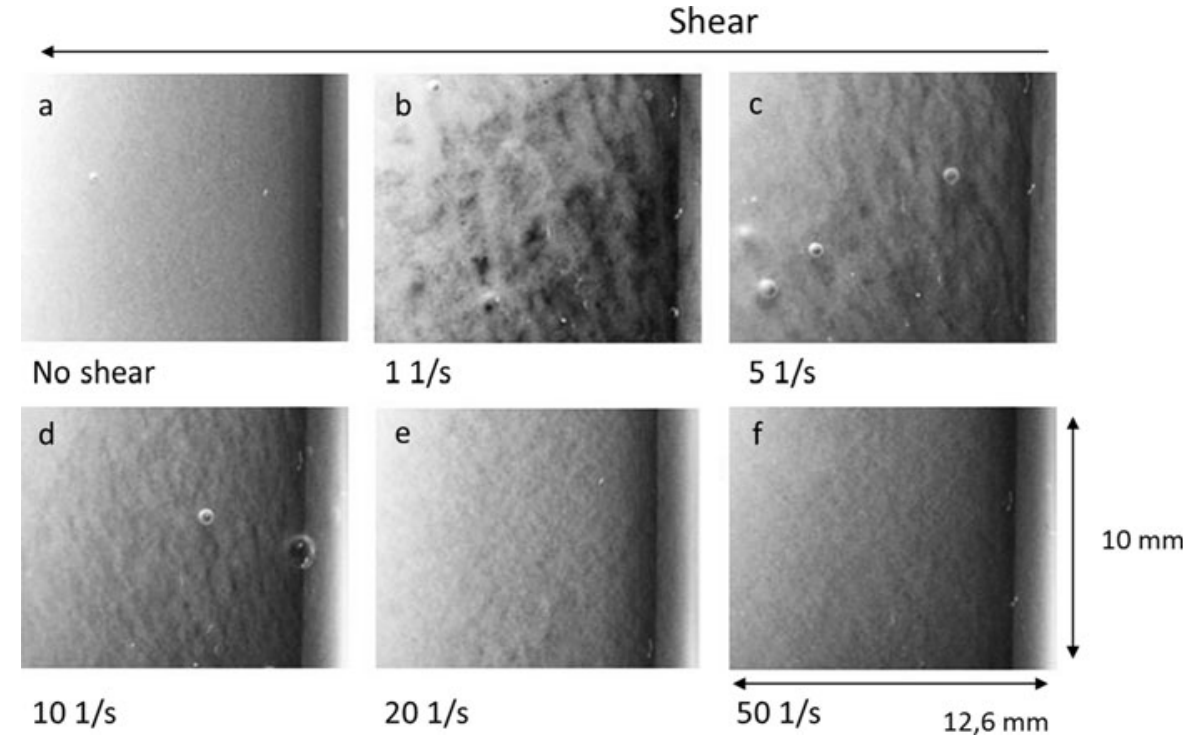

partially attached in the direction parallel to the greatest compression (Fig. 5c, $\dot{\gamma}=5 \mathrm{~s}^{-1}$ ). With increasing shear rate, these floc chains start to buckle and their dimensions decrease (Fig. $5 \mathrm{~d}, \dot{\gamma}=10 \mathrm{~s}^{-1}$ ), finally decomposing into separate, spherical flocs (Fig. 5e, $\dot{\gamma}=20 \mathrm{~s}^{-1}$ ). When the shear rate is increased even further, the size of these spherical flocs decreases (Fig. 5f, $\dot{\gamma}=50 \mathrm{~s}^{-1}$ ). These findings are well in line with those of Björkman (2000).

The effect of measuring geometry gap and material

Rheological tests for fibre suspensions are always dependent on the geometry (Barnes 2000; Björkman 2006; Saarinen et al. 2009). We used two different measuring gaps with regard to glass geometries, as well as two different geometry wall materials, glass and stainless steel.

\section{Gap and wall depletion}

The measuring gap not only limits the maximum size flocs can achieve under any experimental conditions, but it is also related to wall depletion ("wall slip") phenomenon at the geometry boundaries (Barnes 1995). Wall depletion is the removal of solid particles from a certain distance around the geometry boundary leaving a layer of low viscosity suspending medium in their place. Thus, when the suspension is sheared, the actual shear rate is higher near the boundary than in 
the bulk of the suspension leaving the observer with an impression of lower than actual viscosity. The thicker the particles in relation to the geometry gap, the thicker the particle free layer at the boundary and the more it affects the results (Barnes 1995). Therefore, given constant suspension characteristics, the wall depletion should manifest more apparently in results gathered with the smaller gap $(0.9 \mathrm{~mm})$.

Storage and loss moduli were followed in a small amplitude time sweep measurement of four times fluidised sample during the $1 \mathrm{~h}$ recovery period after the preshear mixing period at $\dot{\gamma}=200 \mathrm{~s}^{-1}$ (Fig. 7). With the two different glass geometries, the $\mathrm{G}^{\prime \prime}$ were at the same level, but with the $0.9 \mathrm{~mm}$ gap, the $\mathrm{G}^{\prime}$ was lowered in comparison to the $1.3 \mathrm{~mm}$ gap. This is likely a consequence of more wall depletion with the smaller gap. The stainless steel geometry yields $\mathrm{G}^{\prime}$ levels similar to the larger gap glass geometry, but lower $\mathrm{G}^{\prime \prime}$ levels. This could be explained by the glass surface having a negative, repulsive charge due to -OH groups (Behrens and Grier 2001), and thus inducing a thicker water layer at the boundary. The floc sizes in the preshear were similar in both glass geometries despite the gap difference. The higher shear rates lead to smaller floc sizes, which in turn are less affected by the gap size. Figure 9 shows the average floc diameters for the sample suspension at rest after $59 \min \left(\dot{\gamma}=0 \mathrm{~s}^{-1}\right)$.

Figure 8 presents the peak hold measurement curves for the four times fluidised sample with 0.9 and $1.3 \mathrm{~mm}$ gaps. Part A contains the results with increasing shear rate from 1 to $50 \mathrm{~s}^{-1}$ and Part B the reverse. The floc sizes presented in Fig. 9 correspond to each shear rate, every value a product of measured floc diameter in $\mathrm{x}$ and $\mathrm{y}$ direction. The floc size results are averages of two measurements.

\section{General behaviour in floc size at varying shear rate}

Comparing shear rate data to measured average floc size revealed a correlation between shear rate and floc size depending on geometry gap. Increasing the shear rate from rest (Fig. 8, Part A), first increased the observed floc sizes (Fig. 9) due to formation of floc chains and then decreased when the formed chains broke down and the individual, spherical flocs diminished. The process is reversible back towards low shear rates (Fig. 8, Part B) showing a deviation at

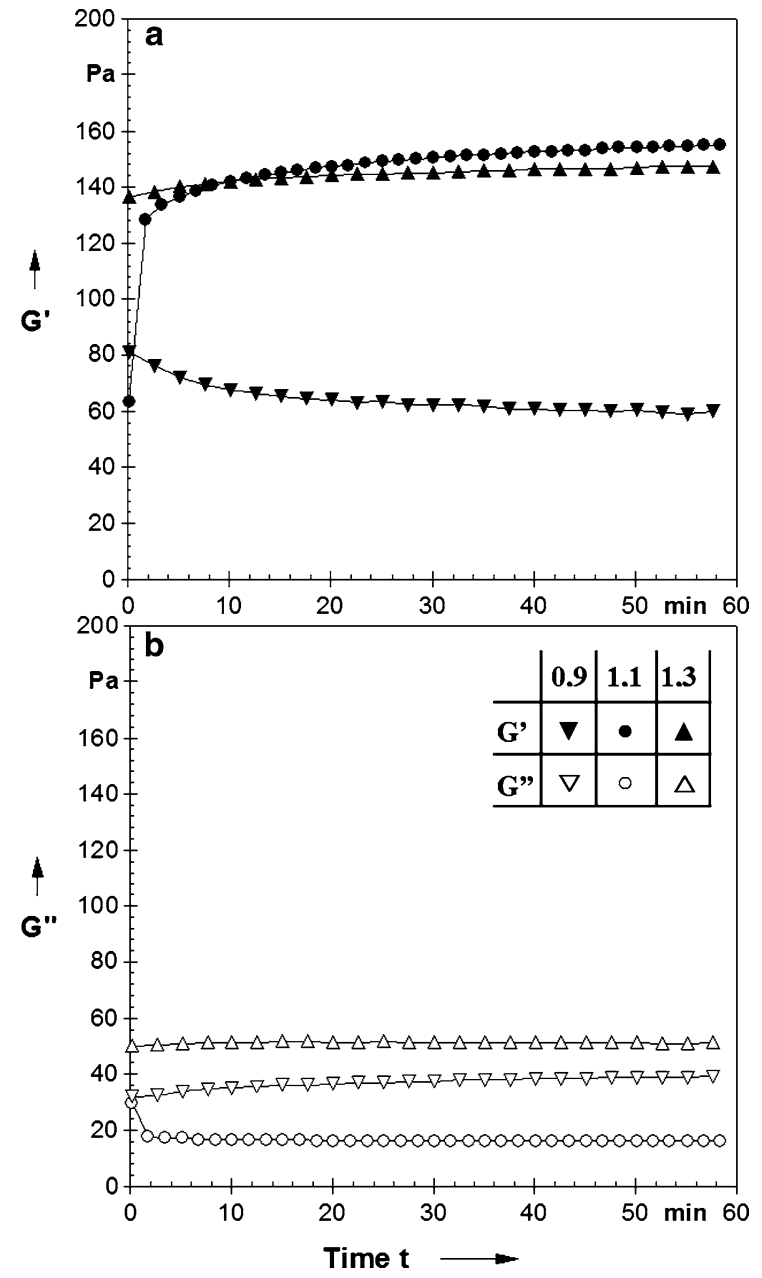

Fig. 7 One hour stabilisation period prior to the peak hold measurements. 1. a storage modulus G' and b loss modulus G' as function of time were observed with three different geometries, stainless steel (gap $1.1 \mathrm{~mm}$, filled circle, open circle), glass 1 (gap $0.9 \mathrm{~mm}$, filled inverted triangle, open inverted triangle), and glass 2 (gap $1.3 \mathrm{~mm}$, filled triangle, open triangle). Temperature $25{ }^{\circ} \mathrm{C}$. The picture was taken after $59 \mathrm{~min}$. Corresponding average floc sizes are presented in Fig. 9 (shear rate 0 ). The sample was fluidised four times

$\dot{\gamma}=5 \mathrm{~s}^{-1}$ and $\dot{\gamma}=1 \mathrm{~s}^{-1}$. We have two alternative explanations for the observed behaviour. First, the lower the shear rate, the longer the time required for the structure to evolve towards a steady state, which is also seen in the shear stress value. Part B generally has lower shear stress from 5 to $1 \mathrm{~s}^{-1}$, which is due to the broader distribution of floc sizes. Second, higher shear rates might also cause irreversible changes (aggregation) to the suspensions network structure and therefore shear stress at a given shear rate would decrease. 
Fig. 8 Peak hold curves for four times fluidised suspension measured with three different geometries, stailess steel cup, gap $1.1 \mathrm{~mm}$ (filled circle), glass cup with gap $0.9 \mathrm{~mm}$ (open inverted triangle), and glass cup with gap $1.3 \mathrm{~mm}$ (open triangle). Shear stress $(\mathrm{Pa})$ is presented as function of time (min) at different shear rates $\left(\mathrm{s}^{-1}\right)$, linear scale. Picture of each state was taken after 9 min $55 \mathrm{~s}$ at each shear rate. Corresponding average floc areas are presented in Fig. 9

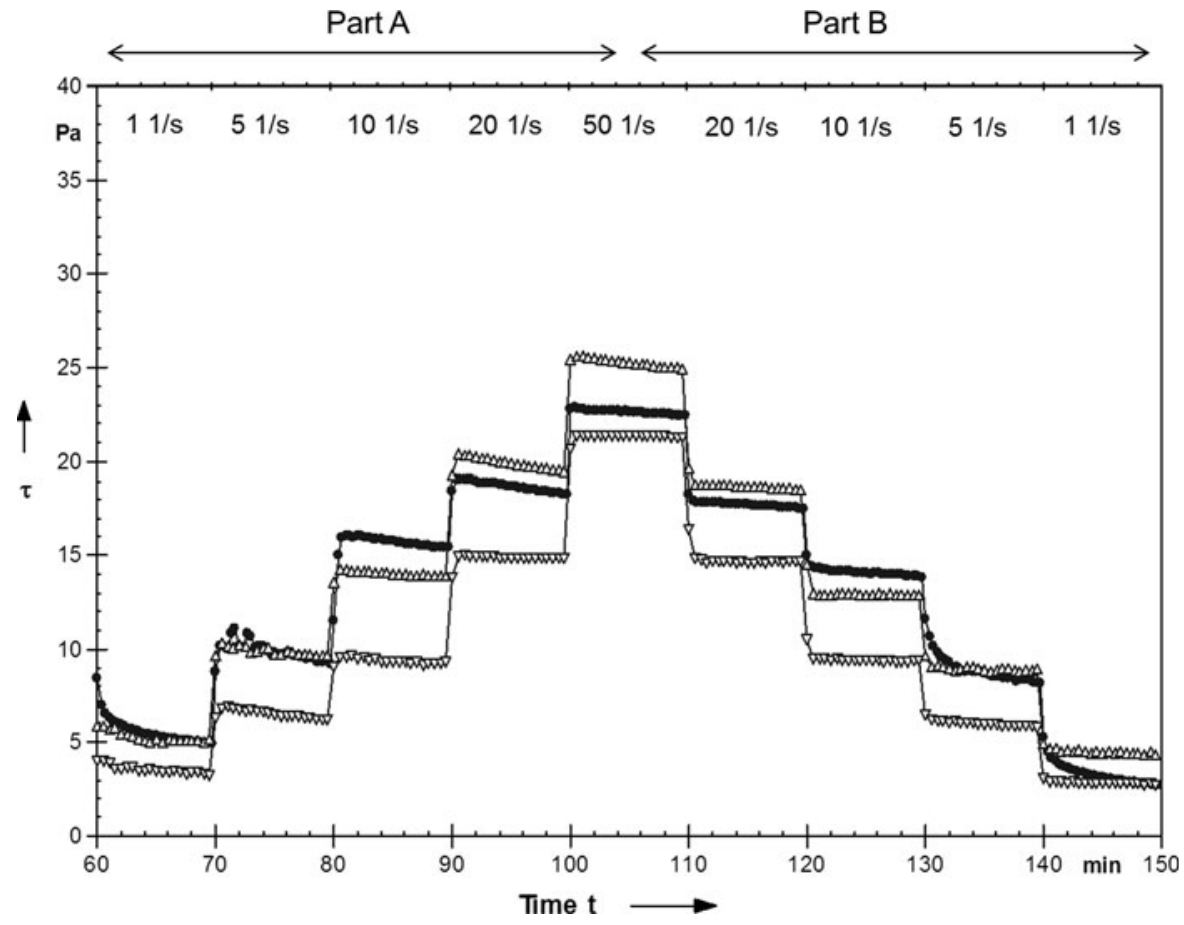

\section{Changing gap and degree of fibrillation versus average floc size}

Figure 9 presents the influences of gap changes on floc diameter averages at different shear rates. The starting point reflects the situation after the preshear interval, which resulted in small average floc size. Starting from rest, the bonds between the existing spherical flocs break down at their weakest points upon subjection to shearing and the liberated flocs begin moving into chain formations. At this point, an increase in width of floc chains is seen in both 1.3 and 0.9 mm gaps. When the shear rate is increased, the floc chains break up and the fibrils start to move in individual, spherical flocs. The spherical floc size decreases monotonically from 20 to $50 \mathrm{~s}^{-1}$. At all shear rates, the apparent floc size is smaller with the $1.3 \mathrm{~mm}$ gap than with the $0.9 \mathrm{~mm}$ gap. This, together with the higher shear stresses with $1.3 \mathrm{~mm}$ gap, again suggest that the stress transfer with the smaller gap was worse than with the larger gap, i.e. $0.9 \mathrm{~mm}$ gap was more susceptible to wall depletion, thus hindering the breakdown of the flocs. Differences in floc sizes and measured shear stresses at a given shear rate even out at higher shear rates, signalling a lessening dependency on geometry boundaries. At higher shear rates, when the flocs become smaller, they are less affected by the gap size. 
The degree of fibrillation affects the floc size (Fig. 10) and rheological response (Fig. 11). The average floc sizes are greater with the less fibrillated
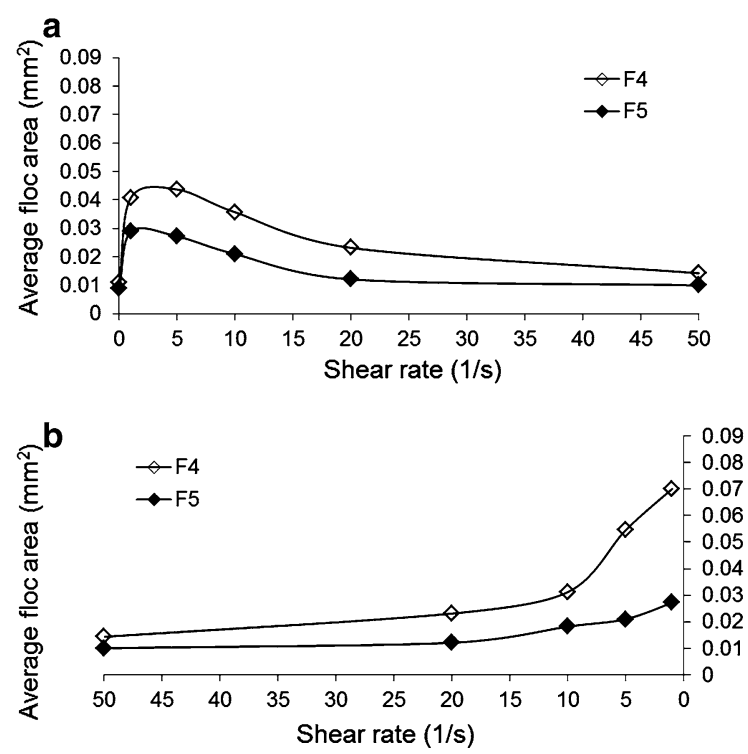

Fig. 10 The effect of the degree of fibrillation on the average floc size. F4 and F5 have been fluidised four and five times. Part A represents the increasing shear rates between 1 and $50 \mathrm{~s}^{-1}$ and Part $\mathrm{B}$ the return from 50 to $1 \mathrm{~s}^{-1}$. The shearing time at each shear rate is $10 \mathrm{~min}$ sample and the shear stresses in steady shear flow smaller. These findings are in line with the increased surface area per volume of F5 compared to F4, which in turn increases the interactions between fibril surfaces.

\section{Effect of NaCl-concentration}

Varying the ionic strength of the suspending medium adjusts the balance between repulsive and attractive forces between the fibrils. By screening the electric charges, fibrils can be brought closer together, strengthening the connections between the fibril contact points. At the same time, the higher the concentration of added ions, the increasing probability for permanent microfibril aggregation, as stated by DLVO theory of colloidal stability (after Deryagin and Landau (1941), and Verwey and Overbeek (1948)). This collapse of the microfibril network leads to a loss of homogeneous gel structure. Furthermore, higher ionic strengths affect to the stability of the suspension under shear. With higher ionic strength, a lesser shear rate suffices to exceed the stabilising effect of the surface charge of the microfibrils, thus inducing permanent aggregation.
Fig. 11 The effect of the degree of fibrillation on the steady shear shear stress (Pa). F4 and F5 have been fluidised four and five times. Part A represents the increasing shear rates between 1 and $50 \mathrm{~s}^{-1}$ and Part B the return from 50 to $1 \mathrm{~s}^{-1}$. The shearing time at each shear rate is $10 \mathrm{~min}$

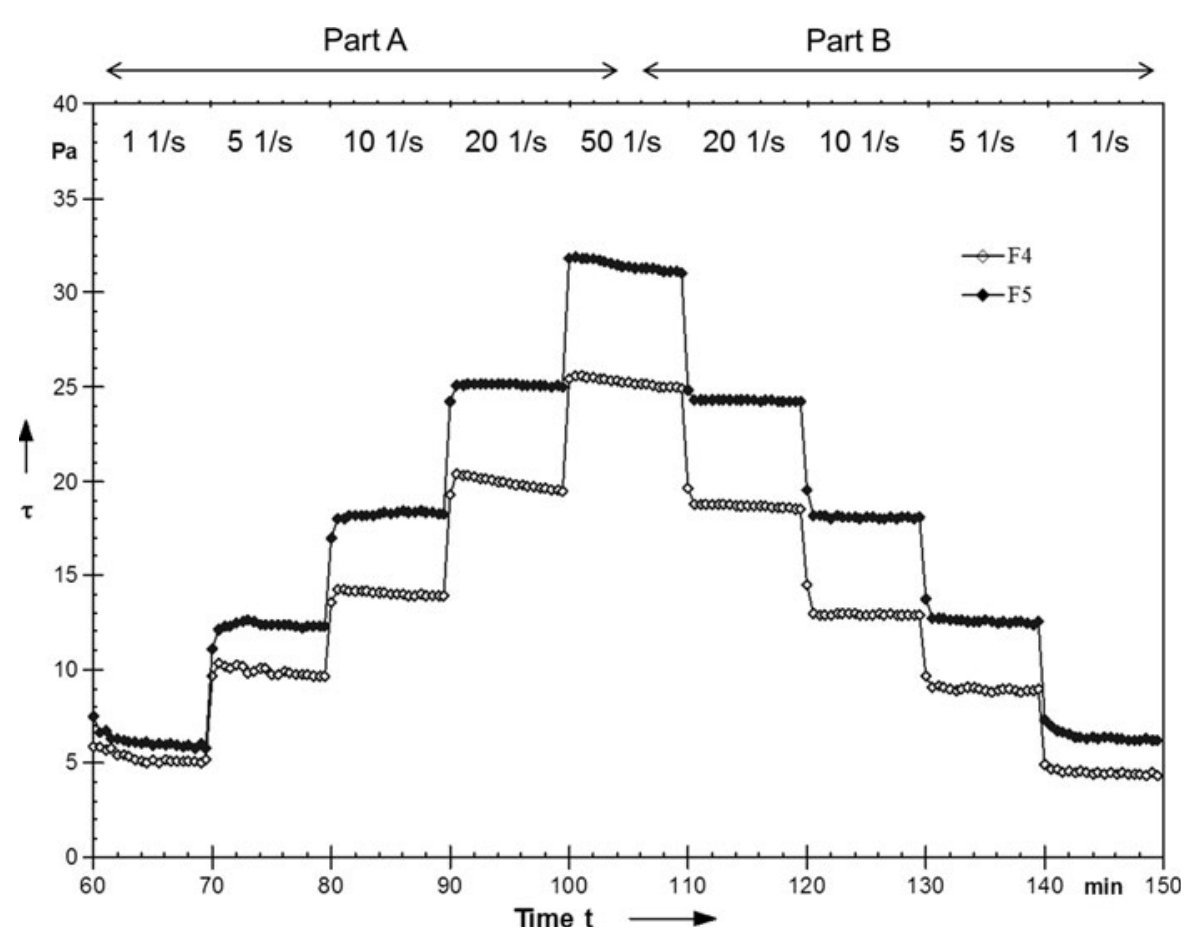




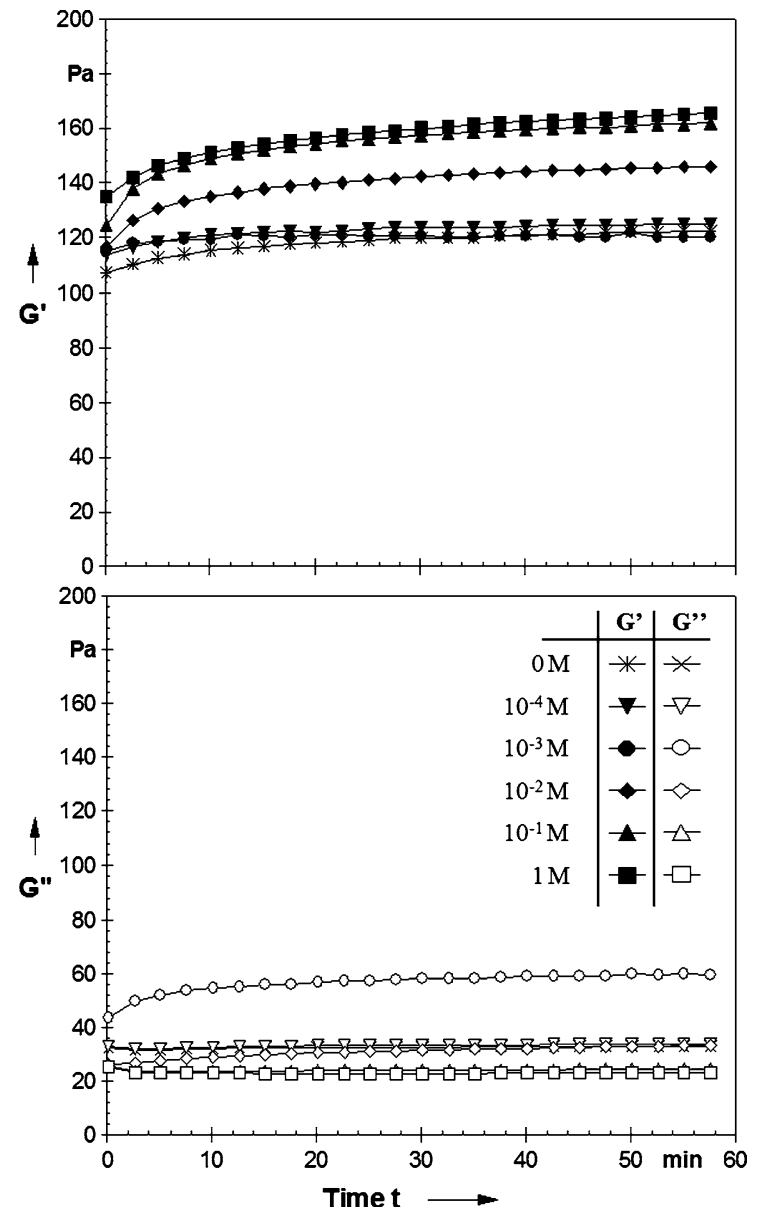

Fig. 12 One hour stabilisation and recovery period for the $\mathrm{NaCl}$ containing suspensions before the start of flow measurement. a Storage modulus G' (Pa) b Loss modulus G', (Pa) as function of time (min). Temperature $25{ }^{\circ} \mathrm{C}$ and the samples were fluidised five times

We tested the rheological properties of MFC suspensions in different $\mathrm{NaCl}$ concentrations. The interpretation of these results is not straightforward due to issues arising from aggregation and wall depletion, the more severe the higher the ionic strength. Recorded images, however, provide the necessary information for correctly interpreting the rheological data.

Figure 12 shows the moduli levels at rest prior to the flow measurements during the $1 \mathrm{~h}$ recovery period. We added varying amounts of $\mathrm{NaCl}$ to the suspensions. During this period the structure of the suspensions stabilise, regardless of reaching a true steady state value, especially with a higher salt content as seen from the slopes of the moduli curves. A small amount of added $\mathrm{NaCl}$ is expected to increase the $\mathrm{G}^{\prime}$ to some extent (Lowys et al. 2001), due to a moderate electrostatic screening effect allowing the fibrils to come into slightly closer contact and increasing the interfibrillar friction. On the other hand, higher $\mathrm{NaCl}$ concentrations result in the energy barrier collapse and aggregation of the fibrils (Ono et al. 2004), as seen in Fig. 13. The aggregation leads to uneven suspension structure and in a narrow gap rheometer the aggregates, which themselves are stiff and dense, may bridge the gap resulting in elevated moduli levels. This is what we see in Fig. 12: the addition of $\mathrm{NaCl}$ at $10^{-2}$ $\mathrm{M}$ and upwards leads to aggregation of microfibrils.

\section{Steady shear tests}

Figure 14 shows results from peak hold flow measurements for suspensions containing $\mathrm{NaCl}$ with a zero molar MFC suspension as a reference curve. At concentration of $10^{-4} \mathrm{M}$, no considerable change appeared on shear stress versus time compared with the unsalted reference. Distinct changes are seen, however, when $\mathrm{NaCl}$ concentration is increased. Adding $10^{-3} \mathrm{M} \mathrm{NaCl}$, dramatically decreases shear stress levels at every shear rate. This is due to flocs being compacted and, at higher ionic strengths, increasingly aggregated with a corollary of an increased amount of free water and thus lower shear stress.
Fig. 13 Microscopic images displaying the aggregation of microfibrils (dark blotches). $\mathrm{NaCl}$ concentrations a $10^{-2} \mathrm{M}$ b $10^{-1} \mathrm{M}$ and c $1 \mathrm{M}$. Scale bar $200 \mu \mathrm{m}$. All samples were fluidised five times
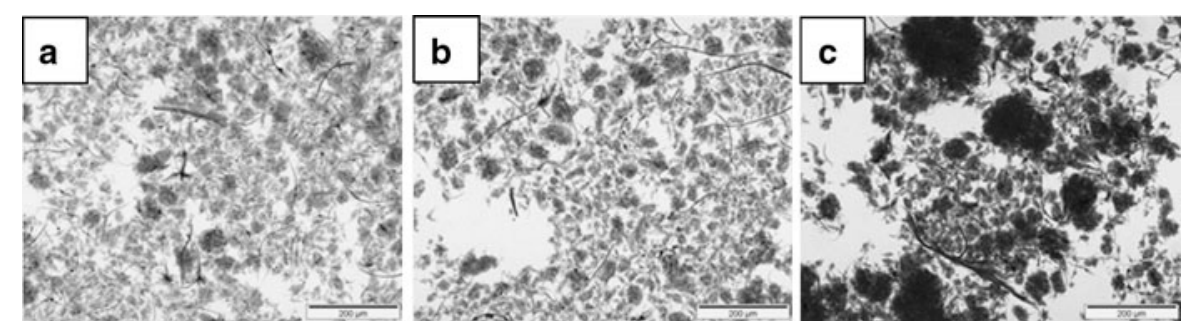
Fig. 14 The effects of $\mathrm{NaCl}$ concentration in peak hold measurements. Shear stress (Pa) as a function of time (min) in different shear rates $\left(\mathrm{s}^{-1}\right)$. An image of the suspension was taken after 9 min 55 s of each peak hold step. The samples were fluidised five times

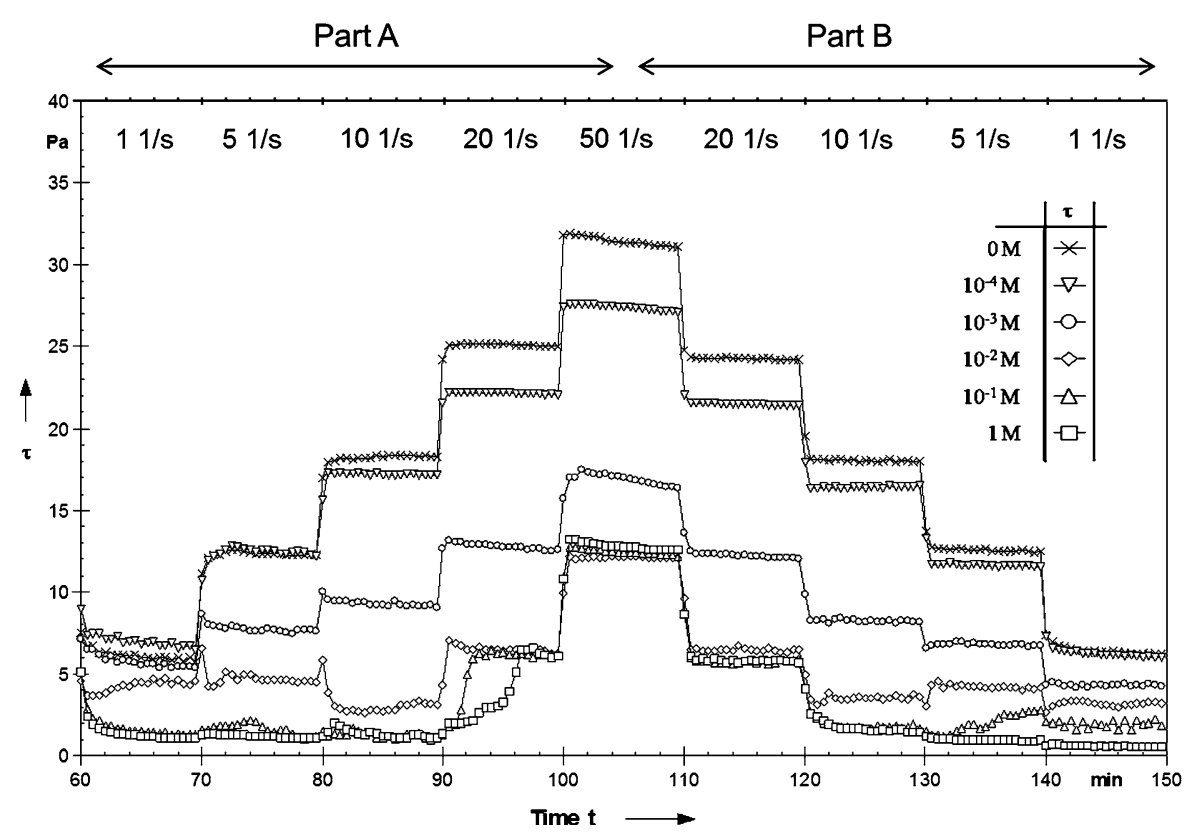

When $\mathrm{NaCl}$ concentration is increased further to $10^{-2} \mathrm{M}$ and above, severe wall depletion starts to show in the results under shear rates of $201 / \mathrm{s}$. Even at 20 1/s (Part A), it takes several minutes before $1 \mathrm{M}$ sample is sheared throughout the complete sample volume in the gap. The salted suspensions are markedly shear thinning, and a layer of low viscous material easily forms near the rotating inner geometry. The more salt added, the higher the apparent shear rate needs to be to overcome the wall depletion and for the complete suspension volume in the gap to be sheared (Barnes 1995). This is due to the denser, partially aggregated flocs, which are harder to break apart. This effect is demonstrated in pictures taken during the flow. Comparing the higher ionic strength sample (Fig. 15) to the unsalted reference (Fig. 5), with salt the network structure does not break up uniformly and gradually, but ruptures suddenly with large voids appearing in the image. Table 3 lists how the shear rate at which the voids appear increases with the ionic strength. The rupture precedes the end of severe wall depletion regime discussed with regard to Fig. 14. Considering this, the observed floc size does not reflect the true floc size in the bulk of the suspension until after the whole suspension volume is under constant shear. However, once the suspension is flowing, disintegration of floc structure is analogous to that of unsalted reference sample. From 20 to 50 1/s, floc size is inversely proportional to the shear rate (Fig. 16),

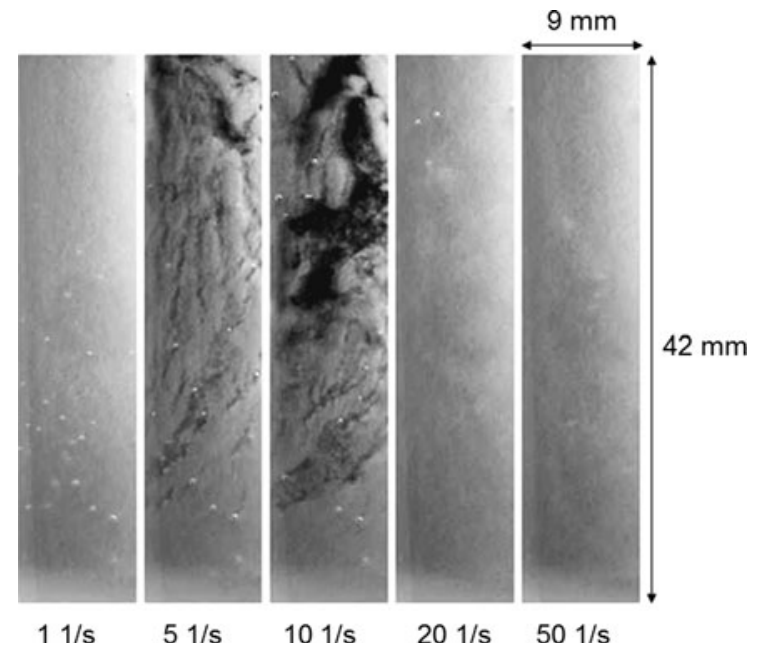

Fig. 15 Example of visible network structure break down with $\mathrm{NaCl}$ concentration of $10^{-1} \mathrm{M}$ seen during the peak hold measurement. Pictures were taken after $9 \min 55 \mathrm{~s}$ at each shear rate and the sample was fluidised five times

Table 3 Changes in suspension structure when $\mathrm{NaCl}$ concentration is increased

\begin{tabular}{ll}
\hline $\mathrm{NaCl}$ Molarity & Changes in structure \\
\hline $10^{-4} \mathrm{M}$ & No rupture \\
$10^{-3} \mathrm{M}$ & No rupture \\
$10^{-2} \mathrm{M}$ & Rupture at shear rate $\left(1 \mathrm{~s}^{-1}\right)$ \\
$10^{-1} \mathrm{M}$ & Rupture at shear rate $\left(5 \mathrm{~s}^{-1}\right)$ \\
$1 \mathrm{M}$ & Rupture at shear rate $\left(10 \mathrm{~s}^{-1}\right)$ \\
\hline
\end{tabular}



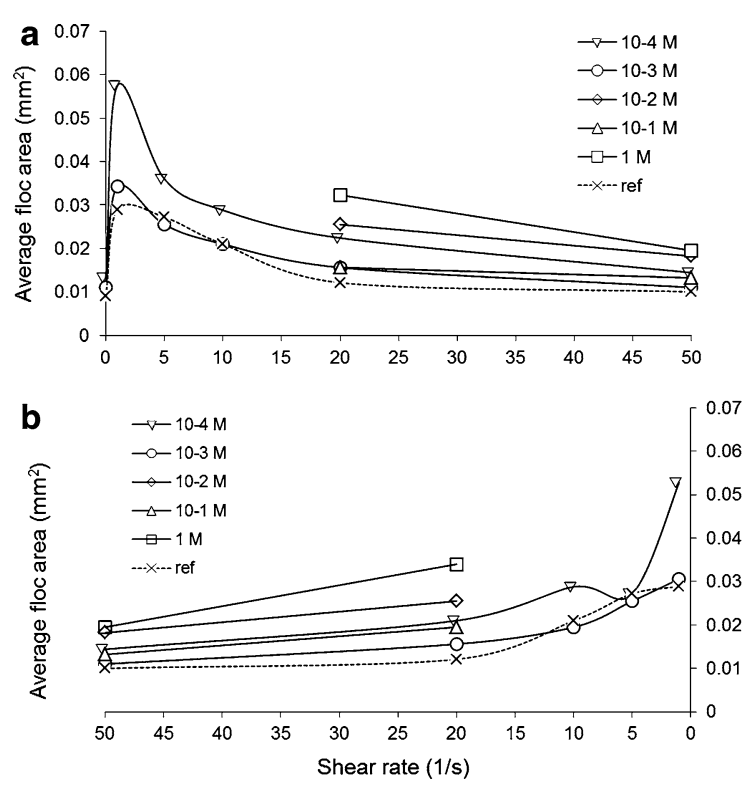

Fig. $16 \mathrm{NaCl}$ concentration's influence to apparent average floc size when shear rate is increased. The samples were fluidised five times

as is to be expected (Björkman 2005). Compared to the unsalted reference, floc sizes are also larger with the addition of salt.

\section{Conclusions}

During the characterisation of microfibrillated cellulose water suspensions, rheological measurements were supplemented by visual examination of the floc structure. This allowed us to directly verify the flocculated nature of the material and observe the various stages of the flow when the flocculated network structure was broken down starting from rest and moving towards constant flow at elevated shear rate. Based on our findings the following conclusions can be drawn:

At rest, MFC water suspensions form a gel structure consisting of sintered flocs. Upon shearing, this flocculated network structure first deforms elastically, and after a certain limit the flocs break loose from each other. At low shear rates, the loosened flocs flow in a chain-like formation and at higher rates, the floc chains buckle and then separate into individual, spherical flocs. The individual spherical floc size is inversely proportional to the shear rate. Therefore, the decomposition of micro scale MFC network in a water suspension follows the same general behaviour as documented for macroscopic fibre suspensions.

Geometry gap dimensions and material affect the flow of flocculated suspensions. A greater gap size corresponded to a lower average floc size at any given shear rate. This was likely caused by greater wall depletion with the smaller gap hindering the breakdown of the flocs. The transparent geometries were constructed from glass and their surface has a dense population of $\mathrm{OH}$ groups. This contributed to the increased wall depletion. The material choices and dimensions in the flow geometries, pipes or pumps, may play an important role in the production processes due to their connection with the energy consumption and suspension homogeneity via the floc structure.

The degree of fibre disintegration into microfibrils depends on the number of passes the suspension has been through the fluidiser. We drew comparisons from suspensions fluidised between four and five times. The four times fluidised sample had a coarser floc structure at any given shear rate. The corresponding shear stresses were also lower.

Ions in the suspending medium greatly affect the flocculation and as a result, the rheological behaviour of MFC suspensions. Adding sodium chloride decreases inter fibrillar repulsion and encourages aggregation and wall depletion. The fibrils remain in flocculated form, and the flocs are larger than without salt. The breakdown of the flocculated network structure in steady shear is the more abrupt the higher the ionic strength, with large fibril free voids appearing in the structure parallel to the direction of greatest compression.

Acknowledgments This work was a part of the Nanosellu I-project established by the Finnish Centre for Nanocellulosic Technologies. Teija Jokila, VTT Technical Research Centre of Finland, is gratefully acknowledged for preparing the sample suspensions. Anni Karppinen, Aalto University, is thanked for valuable observations and discussions regarding the manuscript.

\section{References}

Abe K, Iwamoto S, Yano H (2007) Obtaining cellulose nanofibers with a uniform width of $15 \mathrm{~nm}$ from wood. Biomacromolecules 8:3276-3278

Agoda-Tandjawa G, Durand S, Berot S, Blassel C, Gaillard C, Garnier C, Doublier J-L (2010) Rheological characterization of microfibrillated cellulose suspensions after freezing. Carbohydr Polym 80:677-686 
Barnes HA (1995) A review of the slip (wall depletion) of polymer solutions, emulsions and particle suspensions in viscometers: its cause, character, and cure. J Non-Newton Fluid Mech 56:221-251

Barnes HA (1997) Thixotropy—a review. J Non-Newton Fluid Mech 70:1-33

Barnes HA (2000) Measuring the viscosity of large-particle (and flocculated) suspensions-a note on the necessary gap size of rotational viscometers. J Non-Newton Fluid Mech 94:213-217

Behrens SH, Grier DG (2001) The charge of glass and silica surfaces. J Chem Phys 115:6716-6721

Björkman U (2000) flow of flocculated fibers, 2nd edn. TS Högskoletryckeriet, Stockholm

Björkman U (2003a) Break-up of suspended fibre networks. Nord Pulp Pap Res J 18:32-37

Björkman U (2003b) Stress generation and transmission in suspended fibre networks. Nord Pulp Pap Res J 18:38-43

Björkman U (2005) Floc dynamics in flowing fibre suspensions. Nord Pulp Pap Res J 20:247-252

Björkman U (2006) The metarheology of crowded fibre suspensions. Annu Trans Nord Rheol Soc 14:69-78

Chen B, Tatsumi D, Matsumoto T (2002) Floc structure and flow properties of pulp fiber suspensions. J Soc Rheol Jpn 30:19-25

Deryagin B, Landau L (1941) Theory of the stability of strongly charged lyophobic sols and of the adhesion of strongly charged particles in solutions of electrolytes. Acta PhysicoChimica URSS 14:633-662

Goussé C, Chanzy H, Cerrada ML, Fleury E (2004) Surface silylation of cellulose microfibrils: preparation and rheological properties. Polymer 45:1569-1575

Henriksson M, Henriksson G, Berglund LA, Lindström T (2007) An environmentally friendly method for enzyme-assisted preparation of microfibrillated cellulose (MFC) nanofibers. Eur Polym J 43:3434-3441

Herrick FW, Casebier RL, Hamilton JK, Sandberg KR (1983) Microfibrillated cellulose: morphology and accessibility. J Appl Polym Sci Symp 37:797-813

Hill RJ (2008) Elastic modulus of microfibrillar cellulose gels. Biomacromolecules 9:2963-2966

Hubbe MA (2007) Flocculation and redispersion of cellulosic fiber suspension: a review of effects of hydrodynamic shear and polyelectrolytes. Bioresources 2:296-331

Hubbe MA, Rojas OJ (2008) Colloidal stability and aggregation of lignocellulosic materials in aqueous suspension: a review. Bioresources 3:1419-1491

Iotti M, Gregersen OW, Moe S, Lenes M (2011) Rheological studies of microfibrillar cellulose water dispersions. J Environ Polym Degr 19:137-145

Karema H, Salmela J, Tukiainen M, Lepomäki H (2001) Prediction of paper formation by fluidisation and reflocculation experiments, FRC symposium on the science of papermaking, 17-21 Sept. pp 29
Lasseuguette E, Roux D, Nishiyama Y (2008) Rheological properties of microfibrillar suspension of TEMPO-oxidized pulp. Cellulose 15:425-433

Lowys M-P, Desbrières J, Rinaudo M (2001) Rheological characterization of cellulosic microfibril suspensions. Role of polymeric additives. Food Hydrocolloids 15:25-32

Mewis J, Wagner NJ (2009a) Current trends in suspension rheology. J Nonnewton Fluid Mech 157:147-150

Mewis J, Wagner NJ (2009b) Thixotropy. Adv Colloid Interface Sci 147-148:214-227

Ono H, Shimaya Y, Hongo T, Yamane C (2001) New aqueous dispersion of cellulose sub-micron particles: preparation and properties of transparent cellulose hydrogel(TCG). Trans Mater Res Soc Jpn 26:569-572

Ono H, Shimaya Y, Sato K, Hongo T (2004) 1H spin-spin relaxation time of water and rheological properties of cellulose nanofiber dispersion, transparent cellulose hydrogel (TCG). Polym J 36:684-694

Pääkkö M, Ankerfors M, Kosonen H, Nykänen A, Ahola S, Österberg M, Ruokolainen J, Laine J, Larsson PT, Ikkala O, Lindström T (2007) Enzymatic hydrolysis combined with mechanical shearing and high-pressure homogenization for nanoscale cellulose fibrils and strong gels. Biomacromolecules 8:1934-1941

Saarinen T, Lille M, Seppälä J (2009) Technical aspects on rheological characterization of microfibrillar cellulose water suspensions. Annu Trans Nord Rheol Soc 17: $121-128$

Saito T, Nishiyama Y, Putaux J, Vignon M, Isogai A (2006) Homogeneous suspensions of individualized microfibrils from TEMPO-catalyzed oxidation of native cellulose. Biomacromolecules 7:1687-1691

Salmela J, Kataja M (2005) Floc rupture and re-flocculation in turbulent shear flow, advances in paper science and technology, The Pulp And Paper Fundamental Research Society, Cambridge, 11-16 Sept. pp 15

Tatsumi D, Ishioka S, Matsumoto T (2002) Effect of fiber concentration and axial ratio on the rheological properties of cellulose fiber suspensions. J Soc Rheol Jpn 30:27-32

Turbak AF, Snyder FW, Sandberg KR (1983) Microfibrillated cellulose, a new cellulose product: properties, uses, and commercial potential. J Appl Polym Sci Symp 37:815-827

Verwey EJW, Overbeek JTG with the collaboration of van Ness K (1948) Theory of the stability of lyophobic colloids. The interaction of particles having an electric double layer. Elsevier, New York-Amsterdam, $216 \mathrm{p}$

Wågberg L, Winter L, Ödberg L, Lindström T (1987) On the charge stoichiometry upon adsorption of a cationic polyelectrolyte on cellulosic materials. Colloids Surf 27: 163-173

Yano H, Sugiyama J, Nakagaito AN, Nogi M, Matsuura T, Hikita M, Handa K (2005) Optically transparent composites reinforced with networks of bacterial nanofibers. Adv Mater 17:153-155 (Weinheim, Germany) 